\title{
On Time Staggering for Wave Equations
}

\section{J.G. Verwer}

Published online: 7 August 2007

(C) Springer Science+Business Media, LLC 2007

\begin{abstract}
Grid staggering for wave equations is a validated approach for many applications, as it generally enhances stability and accuracy. This paper is about time staggering. Our aim is to assess a fourth-order, explicit, time-staggered integration method from the literature, through a comparison with two alternative fourth-order, explicit methods. These are the classical Runge-Kutta method and a symmetric-composition method derived from symplectic Euler.
\end{abstract}

Keywords Wave equations · Explicit time integration - Staggered time integration · Composite time integration

\section{Introduction}

The research reported here grew out of our interest in a special fourth-order, explicit, timestaggered integration method proposed in $[4,5]$ for partitioned ODE systems

$$
\begin{aligned}
& u^{\prime}=f(t, v), \\
& v^{\prime}=g(t, u),
\end{aligned}
$$

in particular systems representing semi-discrete wave equations. This partitioned form assumes that $f$ is independent of $u$ and that $g$ is independent of $v$, which of course bears a restriction. Still many problems lead to this partitioned form, such as second-order conservative wave equations posed as first-order systems and other conservative problems, for example, the important Maxwell equations.

If explicit time integration is feasible, as typically holds for wave equations without excessively separated time scales, computational efficiency requires a sufficiently large imaginary stability boundary combined with a sufficiently small truncation error, scaled of course

J.G. Verwer $(\bowtie)$

Centrum voor Wiskunde en Informatica (CWI), P.O. Box 94079, 1090 GB Amsterdam, The Netherlands e-mail: Jan.Verwer@cwi.nl 
with the computational costs per time step. Unfortunately, scaled stability along the imaginary axis meets barriers. In particular, the imaginary stability boundary $\beta_{I m}$ of any explicit, consistent, one-step Runge-Kutta (RK) method is bounded by $s-1$, where $s$ denotes the number of RK stages [8, 15, 16]. By exploiting the special partitioned form (1.1), the staggered one-step method proposed in $[4,5]$ circumvents this barrier. Using effectively four stages, this method has

$$
\beta_{\text {Im }}=16^{1 / 3}+32^{1 / 3} \approx 5.69,
$$

so that compared to the fourth-order, four-stage, classical RK method, which has $\beta_{I m}=$ $2 \sqrt{2}$, an improvement by a factor 2 is obtained. In addition, for autonomous linear problems the staggered method has a 16 times smaller leading truncation error. Compared to the classical RK method, the staggered method thus seems quite beneficial for semi-discrete wave problems of the partitioned form (1.1). The purpose of this paper is to examine this in some detail. We will do this by comparing this method not only to the classical RK method, but also to a fourth-order symmetric-composition method derived from symplectic Euler.

The paper is organized as follows. In Sect. 2 we will discuss the main properties of the staggered method which are relevant to our comparison. Likewise, Sect. 3 is devoted to the symmetric-composition method. The classical Runge-Kutta method will not be discussed, as it can be found in nearly any numerical analysis textbook. In the remainder we will refer to this method as ClassicalRK4. In Sect. 4 we will present results of a numerical comparison between the three fourth-order methods applied to a wave equation with prescribed boundary values. As anticipated, in this case all three fourth-order methods do suffer from order reduction. To some extent, this numerical deficiency diminishes the higher order benefit for high-accuracy calculations, although in general the higher consistency order remains worthwhile. Order reduction is now well understood and in Sect. 5 we will present an error analysis which explains this phenomenon for the staggered method. Final remarks and conclusions are found in Sect. 6.

\section{StaggeredLF4}

\subsection{The Integration Formula}

Time staggering for (1.1) means approximating $u$ and $v$ at interlaced time levels, one after the other. Following [5], we choose integer levels $t_{n}$ for $u$ and half-integer levels $t_{n+1 / 2}$ for $v$ for $n=0,1, \ldots$. Level $t_{n}$ denotes time $t_{n}=n \tau$ with constant step size $\tau$. Let $u_{n}$ and $v_{n+1 / 2}$ be the approximation to $u\left(t_{n}\right)$ and $v\left(t_{n+1 / 2}\right)$, respectively. A well-known timestaggered integration method for system (1.1) is the second-order, explicit, staggered leapfrog rule

$$
\begin{gathered}
u_{n+1}=u_{n}+\tau f\left(t_{n+1 / 2}, v_{n+1 / 2}\right), \\
v_{n+3 / 2}=v_{n+1 / 2}+\tau g\left(t_{n+1}, u_{n+1}\right),
\end{gathered}
$$

henceforth called StaggeredLF2. This method thus steps from $\left(u_{n}, v_{n+1 / 2}\right)$ to $\left(u_{n+1}, v_{n+3 / 2}\right)$ with step size $\tau$. The well-known Yee scheme [17] for the Maxwell equations is of this form.

The 4th-order staggered method from [5] follows the same recipe, except that it uses internal stages as with Runge-Kutta methods. There exist two representations, the first one 
being

$$
\begin{aligned}
k_{1} & =\tau f\left(t_{n+1 / 2}, v_{n+1 / 2}\right), \\
k_{2} & =\tau g\left(t_{n}, u_{n}\right), \\
k_{3} & =\tau f\left(t_{n-1 / 2}, v_{n+1 / 2}-k_{2}\right), \\
k_{4} & =\tau g\left(t_{n+1}, u_{n}+k_{1}\right), \\
k_{5} & =\tau f\left(t_{n+3 / 2}, v_{n+1 / 2}+k_{4}\right), \\
u_{n+1} & =u_{n}+\frac{22}{24} k_{1}+\frac{1}{24} k_{3}+\frac{1}{24} k_{5} . \\
e_{1} & =\tau g\left(t_{n+1}, u_{n+1}\right), \\
e_{2} & =\tau f\left(t_{n+1 / 2}, v_{n+1 / 2}\right), \\
e_{3} & =\tau g\left(t_{n}, u_{n+1}-e_{2}\right), \\
e_{4} & =\tau f\left(t_{n+3 / 2}, v_{n+1 / 2}+e_{1}\right), \\
e_{5} & =\tau g\left(t_{n+2}, u_{n+1}+e_{4}\right), \\
v_{n+3 / 2} & =v_{n+1 / 2}+\frac{22}{24} e_{1}+\frac{1}{24} e_{3}+\frac{1}{24} e_{5} .
\end{aligned}
$$

In the formulas defining $u_{n+1}$, odd and even numbered stages are assigned to $f$ and $g$, respectively, while in the formulas defining $v_{n+3 / 2}$ it is the other way around. The approximation $v_{n+3 / 2}$ is defined by the same coefficients as used for $u_{n+1}$, the only difference being that $f$ and $g$ are interchanged and that all time levels in $v_{n+3 / 2}$ are shifted forward with $1 / 2$. The missing value $v_{1 / 2}$ at the start is to be provided by a regular one-step method, such as ClassicalRK4. Note that although (2.2) is written in 5 stages, it can be implemented with 4 evaluations of $f$ and $g$, because $e_{2}=k_{1}$ and $e_{1}$ can be saved to provide $k_{2}$ at the next time step. Hence the computational costs per step are comparable to those of ClassicalRK4 and if the above savings are used, the costs are equal.

The second representation, which reveals its relation with StaggeredLF2, is given by

$$
\begin{aligned}
U_{1} & =u_{n}+\tau f\left(t_{n+1 / 2}, v_{n+1 / 2}\right), \\
V_{2} & =v_{n+1 / 2}-\tau g\left(t_{n}, u_{n}\right), \\
U_{3} & =u_{n}+\tau f\left(t_{n-1 / 2}, V_{2}\right), \\
V_{4} & =v_{n+1 / 2}+\tau g\left(t_{n+1}, U_{1}\right), \\
U_{5} & =u_{n}+\tau f\left(t_{n+3 / 2}, V_{4}\right), \\
u_{n+1} & =\frac{22}{24} U_{1}+\frac{1}{24} U_{3}+\frac{1}{24} U_{5} . \\
V_{1} & =v_{n+1 / 2}+\tau g\left(t_{n+1}, u_{n+1}\right), \\
U_{2} & =u_{n+1}-\tau f\left(t_{n+1 / 2}, v_{n+1 / 2}\right), \\
V_{3} & =v_{n+1 / 2}+\tau g\left(t_{n}, U_{2}\right), \\
U_{4} & =u_{n+1}+\tau f\left(t_{n+3 / 2}, V_{1}\right), \\
V_{5} & =v_{n+1 / 2}+\tau g\left(t_{n+2}, U_{4}\right), \\
v_{n+3 / 2} & =\frac{22}{24} V_{1}+\frac{1}{24} V_{3}+\frac{1}{24} V_{5} .
\end{aligned}
$$

From this representation one sees more clearly that steps in the negative direction are taken, viz., for $V_{2}$ and $U_{2}$. In the remainder we will refer to (2.2) and (2.3) as StaggeredLF4. 
Taylor expanding at $t_{n+1 / 2}$ from exact starting values $u\left(t_{n}\right), v\left(t_{n+1 / 2}\right)$, yields for system (1.1) in autonomous form the local error expression

$$
\begin{aligned}
\delta_{u}= & u\left(t_{n+1}\right)-u_{n+1} \\
= & \tau^{5}\left(\frac{1}{1920} u^{(5)}-\frac{1}{576} f_{j} g_{k l}^{j} f_{m}^{l} g^{k} g^{m}-\frac{1}{576} f_{j} g_{k l m}^{j} f^{k} f^{l} f^{m}\right. \\
& -\frac{1}{288} f_{j} g_{k l}^{j} f^{k} f_{m}^{l} g^{m}-\frac{1}{96} f_{j k} g^{j} g_{l m}^{k} f^{l} f^{m}-\frac{1}{96} f_{j k} g^{j} g_{l}^{k} f_{m}^{l} g^{m} \\
& \left.-\frac{1}{96} f_{j k} g_{l}^{j} f^{l} g_{m}^{k} f^{m}-\frac{1}{48} f_{j k l} g^{j} g^{k} g_{m}^{l} f^{m}-\frac{1}{288} f_{j k l m} g^{j} g^{k} g^{l} g^{m}\right)+\mathcal{O}\left(\tau^{6}\right) .
\end{aligned}
$$

For the $v$-component expanded at $t_{n+1}$ a similar expression exists. For constant coefficient autonomous linear systems (1.1), for which all elementary differentials in this expression vanish, the leading local error term is equal to $\frac{1}{1920} \tau^{5} u^{(5)}$, which is very small for a fourthorder method. This is a clear advantage of time staggering. In the linear case, ClassicalRK4 has as leading local error term $\frac{1}{120} \tau^{5} u^{(5)}$, being 16 times larger. However, for arbitrary nonlinear problems the advantage may diminish, because elementary differentials can be present with error constants significantly larger than $\frac{1}{1920}$.

\subsection{Linear Stability}

For $\lambda \in \mathbb{C}$, stability properties are examined for the 2-component linear test model [5]

$$
\left(\begin{array}{l}
u \\
v
\end{array}\right)^{\prime}=\left(\begin{array}{ll}
0 & \lambda \\
\lambda & 0
\end{array}\right)\left(\begin{array}{l}
u \\
v
\end{array}\right)
$$

The $2 \times 2$-matrix is decomposed as

$$
\left(\begin{array}{ll}
0 & \lambda \\
\lambda & 0
\end{array}\right)=\frac{1}{2}\left(\begin{array}{cc}
1 & -1 \\
1 & 1
\end{array}\right)\left(\begin{array}{cc}
\lambda & 0 \\
0 & -\lambda
\end{array}\right)\left(\begin{array}{cc}
1 & 1 \\
-1 & 1
\end{array}\right) .
$$

Multiplying by $\tau$, denoting $z=\tau \lambda$, and computing the matrix exponential, this decomposition reveals the exact solution expression

$$
\left(\begin{array}{l}
u\left(t_{n+1}\right) \\
v\left(t_{n+1}\right)
\end{array}\right)=\left(\begin{array}{cc}
\cosh (z) & \sinh (z) \\
\sinh (z) & \cosh (z)
\end{array}\right)\left(\begin{array}{l}
u\left(t_{n}\right) \\
v\left(t_{n}\right)
\end{array}\right),
$$

which on the staggered time grid transforms into

$$
\left(\begin{array}{l}
u\left(t_{n+1}\right) \\
v\left(t_{n+3 / 2}\right)
\end{array}\right)=\left(\begin{array}{cc}
1 & 2 \sinh \left(\frac{z}{2}\right) \\
2 \sinh \left(\frac{z}{2}\right) & 1+4 \sinh ^{2}\left(\frac{z}{2}\right)
\end{array}\right)\left(\begin{array}{l}
u\left(t_{n}\right) \\
v\left(t_{n+1 / 2}\right)
\end{array}\right) .
$$

Likewise, applying StaggeredLF4 to the test model gives the numerical recursion

$$
\left(\begin{array}{l}
u_{n+1} \\
v_{n+3 / 2}
\end{array}\right)=\mathcal{M}_{s}\left(\begin{array}{l}
u_{n} \\
v_{n+1 / 2}
\end{array}\right), \quad \mathcal{M}_{s}=\left(\begin{array}{cc}
1 & \beta \\
\beta & 1+\beta^{2}
\end{array}\right),
$$

where $\beta=z+\frac{1}{24} z^{3}$. Note that the StaggeredLF2 method (2.1) gives the same recursion with $\beta=z$. 
Remark 2.1 For $z \rightarrow 0$ the entries of $\mathcal{M}_{s}$ should coincide with the corresponding entries of the matrix in (2.7) up to at least order four. Let $\mathcal{M}_{s e}$ denote this matrix. Then we get

$$
\mathcal{M}_{s e}-\mathcal{M}_{s}=\left(\begin{array}{cc}
0 & \frac{1}{1920} z^{5}+\mathcal{O}\left(z^{7}\right) \\
\frac{1}{1920} z^{5}+\mathcal{O}\left(z^{7}\right) & \frac{1}{860} z^{6}+\mathcal{O}\left(z^{8}\right)
\end{array}\right), \quad z \rightarrow 0,
$$

revealing the leading error constant 1/1920. Similarly, for ClassicalRK4 we have

$$
\begin{gathered}
\left(\begin{array}{c}
u_{n+1} \\
v_{n+1}
\end{array}\right)=\mathcal{M}\left(\begin{array}{c}
u_{n} \\
v_{n}
\end{array}\right), \\
\mathcal{M}=\left(\begin{array}{cc}
1+\frac{1}{2} z^{2}+\frac{1}{24} z^{4} & z+\frac{1}{6} z^{3} \\
z+\frac{1}{6} z^{3} & 1+\frac{1}{2} z^{2}+\frac{1}{24} z^{4}
\end{array}\right),
\end{gathered}
$$

and denoting the matrix in (2.6) by $\mathcal{M}_{e}$ we find

$$
\mathcal{M}_{e}-\mathcal{M}=\left(\begin{array}{cc}
\frac{1}{720} z^{6}+\mathcal{O}\left(z^{8}\right) & \frac{1}{120} z^{5}+\mathcal{O}\left(z^{7}\right) \\
\frac{1}{120} z^{5}+\mathcal{O}\left(z^{7}\right) & \frac{1}{720} z^{6}+\mathcal{O}\left(z^{8}\right)
\end{array}\right), \quad z \rightarrow 0,
$$

revealing the 16 times larger leading error constant.

StaggeredLF4 is called (linearly) stable if $\mathcal{M}_{s}$ is power bounded. This holds, if and only if its two eigenvalues lie on the unit disc and are different when they lie on the unit circle (the root condition). The stability region is then defined by the set of values $z=\tau \lambda$ in $\mathcal{C}$ for which this holds. With $\mu$ denoting an eigenvalue, the characteristic equation of $\mathcal{M}_{s}$ reads $(1-\mu)^{2}=\beta^{2} \mu$. Because the product of the two zeroes is equal to one, we can restrict ourselves to zeroes on the unit circle. Inserting $\mu=e^{i \phi}$ gives for $\beta=z+\frac{1}{24} z^{3}$ the condition $\beta^{2}=4 i^{2} \sin ^{2}(\phi / 2)$. It thus immediately follows that we have stability only if $z$ is purely imaginary.

Hence the stability region of StaggeredLF4 is an interval along the imaginary axis and on this interval the scheme is non-dissipative. A further elementary calculation shows that the single real zero of the cubic equation $a^{3}-24 a-48=0$ determines the imaginary stability boundary

$$
\beta_{\text {Im }}=16^{1 / 3}+32^{1 / 3} \approx 5.69,
$$

defining the imaginary stability interval $\left(-\beta_{I m}, \beta_{I m}\right)$. For $z= \pm i \beta_{I m}$ the two roots of the characteristic equation of $\mathcal{M}_{s}$ coincide, violating the root condition. ClassicalRK4 has $\beta_{I m}=2 \sqrt{2} \approx 2.82$, being a factor 2 smaller. However, the properly scaled imaginary stability boundary of StaggeredLF4, that is $\beta_{I m} / 4 \approx 1.42$, is smaller than the (scaled) $\beta_{I m}$ of StaggeredLF2, which equals 2.0.

Apparently, for the special ODE problem (1.1), which is associated to non-dissipative wave equations, StaggeredLF4 has attractive properties. It improves ClassicalRK4 with a 16 times smaller error constant in the linear case and a 2 times larger $\beta_{\text {Im }}$ for equal costs. It also improves StaggeredLF2 by its order four compared to order two, but at the expense of a four times higher workload per step and a smaller scaled $\beta_{I m}$. Nevertheless, for highaccuracy calculations, as with long-time applications, the higher order will be advantageous. Similar as for StaggeredLF2, a restriction is that even a small amount of dissipation rules out the staggered method, whereas ClassicalRK4 remains applicable. 
Remark 2.2 Test model (2.4) is unstable for any $\lambda$ with a nonzero real part. The staggered methods mimic this, restricting $\lambda$ to be purely imaginary for stability. The $\lambda$ can be thought of as resulting from Fourier-von Neumann analysis and thus representing all eigenvalues in the spectrum of a semi-discrete wave operator. It suffices to work with a single $\lambda$-value. Using two eigenvalues as in

$$
\left(\begin{array}{l}
u \\
v
\end{array}\right)^{\prime}=\left(\begin{array}{cc}
0 & \lambda_{1} \\
\lambda_{2} & 0
\end{array}\right)\left(\begin{array}{l}
u \\
v
\end{array}\right),
$$

makes no difference due to the similarity transformation

$$
\left(\begin{array}{cc}
\sqrt{\frac{\lambda_{2}}{\lambda_{1}}} & 0 \\
0 & 1
\end{array}\right)\left(\begin{array}{cc}
0 & \lambda_{1} \\
\lambda_{2} & 0
\end{array}\right)\left(\begin{array}{cc}
\sqrt{\frac{\lambda_{1}}{\lambda_{2}}} & 0 \\
0 & 1
\end{array}\right)=\left(\begin{array}{cc}
0 & \sqrt{\lambda_{1} \lambda_{2}} \\
\sqrt{\lambda_{1} \lambda_{2}} & 0
\end{array}\right) .
$$

Hence, for stability analysis the two models are equivalent. By interpreting $\lambda, \lambda_{1}, \lambda_{2}$ properly, all conclusions and results derived for (2.4) are also valid for (2.13) and vice-versa.

\section{SymmetricCO4}

To assess the practical use and performance of StaggeredLF4, we will also compare it to an explicit, fourth-order, symmetric-composition method based on the symplectic Euler rule

$$
\begin{aligned}
& u_{n+1}=u_{n}+\tau f\left(t_{n}, v_{n}\right), \\
& v_{n+1}=v_{n}+\tau g\left(t_{n+1}, u_{n+1}\right),
\end{aligned}
$$

and its adjoint

$$
\begin{aligned}
& u_{n+1}=u_{n}+\tau f\left(t_{n+1}, v_{n+1}\right), \\
& v_{n+1}=v_{n}+\tau g\left(t_{n}, u_{n}\right) .
\end{aligned}
$$

\subsection{The Integration Formula}

Composition is a well-known idea within geometric integration, see e.g. [2, 6, 9-11, 13]. Our method is derived as explained in [6, Sect. V.3, formula (3.1)]. It uses a coefficient set due to [9] which is given in formula (3.6) in [6]. Let $\Phi_{\alpha \tau}$ and $\Phi_{\beta \tau}^{*}$ represent (3.1) and (3.2) applied with step size $\alpha \tau$ and $\beta \tau$, respectively. The method then performs a time step from $\left(u_{n}, v_{n}\right)$ to $\left(u_{n+1}, v_{n+1}\right)$, using an overall step of length $\tau$ through the symmetric composition

$$
\Psi_{\tau}=\Phi_{\alpha_{s} \tau} \circ \Phi_{\beta_{s} \tau}^{*} \circ \cdots \circ \Phi_{\alpha_{1} \tau} \circ \Phi_{\beta_{1} \tau}^{*},
$$

where $s=5$ and the $\alpha_{k}, \beta_{k}$ are given by

$$
\begin{aligned}
& \beta_{1}=\alpha_{5}=\frac{14-\sqrt{19}}{108}, \quad \alpha_{1}=\beta_{5}=\frac{146+5 \sqrt{19}}{540}, \\
& \beta_{2}=\alpha_{4}=\frac{-23-20 \sqrt{19}}{270}, \quad \alpha_{2}=\beta_{4}=\frac{-2+10 \sqrt{19}}{135}, \quad \beta_{3}=\alpha_{3}=\frac{1}{5} .
\end{aligned}
$$

Introduce for $k=1, \ldots, 5$ the coefficients $a_{k}=\alpha_{1}+\cdots+\alpha_{k}$ and $b_{k}=\beta_{1}+\cdots+\beta_{k}$. Further introduce $\alpha_{0}=0, a_{0}=0, b_{0}=0$ and $U_{0}=u_{n}, V_{0}=v_{n}$. The composition $\Psi_{\tau}$ then can be 
economically and compactly written in the form

$$
\begin{aligned}
V_{k} & =V_{k-1}+\left(\beta_{k}+\alpha_{k-1}\right) \tau g_{k-1} \\
U_{k} & =U_{k-1}+\left(\beta_{k}+\alpha_{k}\right) \tau f_{k} \\
v_{n+1} & =V_{5}+\alpha_{5} \tau g\left(t_{n+1}, u_{n+1}\right), \\
u_{n+1} & =U_{5},
\end{aligned}
$$

where

$$
\begin{aligned}
g_{k-1} & =g\left(t_{n}+\left(b_{k-1}+a_{k-1}\right) \tau, U_{k-1}\right), \\
f_{k} & =f\left(t_{n}+\left(b_{k}+a_{k-1}\right) \tau, V_{k}\right) .
\end{aligned}
$$

Because $g\left(t_{n+1}, u_{n+1}\right)$ can be saved, in this form the method requires 5 evaluations of $f$ and $g$ per step, just one more than ClassicalRK4 and StaggeredLF4. In the remainder we will refer to (3.5) as SymmetricCO4. Like StaggeredLF4, also this method takes substeps in the negative direction.

\subsection{Linear Stability}

When applied to test model (2.4), SymmetricCO4 yields the recurrence

$$
\left(\begin{array}{l}
u_{n+1} \\
v_{n+1}
\end{array}\right)=\mathcal{M}\left(\begin{array}{l}
u_{n} \\
v_{n}
\end{array}\right), \quad \mathcal{M}=\prod_{k=5}^{1} \mathcal{M}_{k},
$$

where

$$
\mathcal{M}_{k}=\left(\begin{array}{cc}
1+\beta_{k}\left(\alpha_{k}+\beta_{k}\right) z^{2} & \left(\alpha_{k}+\beta_{k}\right) z \\
\left(\alpha_{k}+\beta_{k}\right)\left(z+\alpha_{k} \beta_{k} z^{3}\right) & 1+\alpha_{k}\left(\alpha_{k}+\beta_{k}\right) z^{2}
\end{array}\right) .
$$

The entry $\mathcal{M}(1,2)$ approximates $\sinh (z)$ in $(2.6)$ with leading error $\mathcal{O}\left(z^{5}\right)$ and constant $\approx 1 / 2121$, which is somewhat smaller than the corresponding constant $1 / 1920$ of StaggeredLF4.

We have examined power boundedness of $\mathcal{M}$ experimentally. Like StaggeredLF4, SymmetricCO 4 is unstable for any $\lambda$ with a nonzero real part. For its imaginary stability boundary $\beta_{I m}$, we found 3.0, approximately, being $5.69 / 3 \approx 1.9$ smaller than for StaggeredLF4. When comparing the scaled imaginary stability boundaries

$$
\begin{array}{ll}
2 \sqrt{2} / 4 & \approx 0.71 \text { ClassicalRK4 } \\
\left(16^{1 / 3}+32^{1 / 3}\right) / 4 & \approx 1.42 \text { StaggeredLF4 } \\
3.0 / 5 & \approx 0.60 \text { SymmetricCO4 }
\end{array}
$$

it is clear that StaggeredLF4 is most promising in situations where the step size is truly limited by stability. Of course, then the second-order staggered LF rule (2.1) remains a strong competitor, as it has $\beta_{\text {Im }}=2.0$ and uses just one $f$ and $g$ per step.

Remark 3.1 There exist several coefficient sets for symmetric-composition methods of order four [6]. For our assessment of StaggeredLF4, the scaled imaginary stability boundary is important, but also the accuracy. The coefficients (3.4) due to McLachlan [9] are known to give particularly small error terms. As a possible alternative we have computed the boundary for 
the unique coefficient set for $s=3$, see [6]. We found 1.57/3, which is smaller than 5.0/6. Moreover, the $s=3$ formula is known to lead to significantly lower accuracies. As a second alternative we have examined coefficients for $s=6$ due to [2], which are also advocated in [6], formula (3.7), for giving small error terms. For these coefficients we found 3.16/6 as scaled imaginary stability boundary, which is again smaller than $5.0 / 6$. We thus have concluded that the current (geometric) integrator SymmetricCO4 makes a good competitor for StaggeredLF4, despite the fact that StaggeredLF4 does have a substantially larger scaled imaginary stability boundary.

\section{Order Reduction}

Higher order methods using internal stages do suffer from order reduction resulting in loss of accuracy. To a certain extent this is problem dependent, but a rule of thumb is that it happens with inhomogeneous, prescribed boundary data. We will illustrate this numerically for StaggeredLF4 and its two fourth-order competitors. Because with loss of accuracy StaggeredLF2 may come into sight, this second-order method is also included.

Although simple, the 1D linear wave equation

$$
\phi_{t}=\psi_{x}, \quad \psi_{t}=\phi_{x}, \quad 0 \leq x \leq 1,
$$

serves our purpose. As solution we choose

$$
\begin{aligned}
& \phi(x, t)=\frac{1}{2}\left(\phi_{0}(x-t)+\phi_{0}(x+t)\right), \\
& \psi(x, t)=\frac{1}{2}\left(\phi_{0}(x-t)-\phi_{0}(x+t)\right),
\end{aligned}
$$

prescribed by the pulse profile $\phi_{0}(x)=e^{-100\left(x-\frac{1}{2}\right)^{2}}$. See Fig. 1 .

Let $h=1 /(N+1), x_{i}=i h$ for $i=0,1, \ldots, N+1$, and let $u_{i}(t)$ and $v_{i}(t)$ denote the semi-discrete approximations to $\phi\left(x_{i}, t\right)$ and $\psi\left(x_{i}, t\right)$, respectively. We then discretize $\phi_{t}=\psi_{x}$ in space with the fourth-order implicit scheme

$$
\frac{1}{6}\left(u_{i-1}^{\prime}+4 u_{i}^{\prime}+u_{i+1}^{\prime}\right)=\frac{1}{2 h}\left(v_{i+1}-v_{i-1}\right), \quad i=1, \ldots, N .
$$
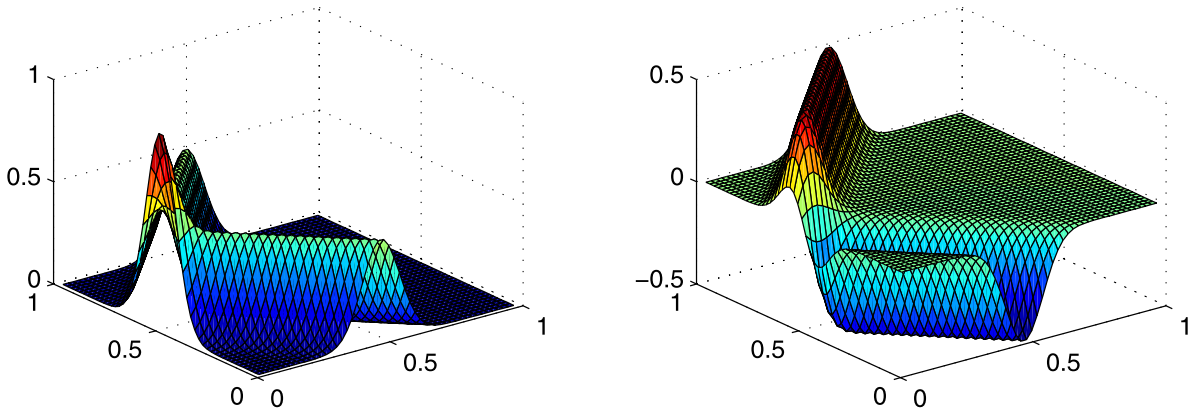

Fig. 1 The exact solution of the 1D test problem (4.1). At the left $\phi$, at the right $\psi$ 
The boundary values $u_{0}^{\prime}, u_{N+1}^{\prime}$ and $v_{0}, v_{N+1}$ are prescribed from the exact solution. ${ }^{1}$ The second equation $\psi_{t}=\phi_{x}$ is semi-discretized similarly.

Arranging the unknowns $u_{i}, v_{i}$ in vectors $u, v$ of length $N$, we then arrive at the inhomogeneous linear systems

$$
\begin{array}{ll}
u^{\prime}=A v+a(t), & A=M^{-1} S, a(t)=M^{-1} s_{u}(t), \\
v^{\prime}=A u+b(t), & A=M^{-1} S, b(t)=M^{-1} s_{v}(t),
\end{array}
$$

where the definitions of the tridiagonal matrices $M, S$ and source terms $s_{u}, s_{v}$ shall be clear. The matrix $A$ does have purely imaginary eigenvalues, but $A$ is not truly skew-symmetric (near the boundary points) as it would be with periodic boundary values. The spectral radius of $A$ equals $1.74 / h$, approximately, resulting in the following maximal step sizes for stability

$$
\tau=\left\{\begin{array}{l}
2 \sqrt{2} h / 1.74 \approx 1.62 h \text { ClassicalRK4 } \\
5.69 h / 1.74 \approx 3.27 h \text { StaggeredLF4 } \\
3.0 h / 1.74 \approx 1.72 h \text { SymmetricCO4 } \\
2.0 h / 1.74 \approx 1.14 h \text { StaggeredLF2 }
\end{array}\right.
$$

With a minor adjustment to hit chosen output times within an integer number of steps, which results in slightly smaller values, the step sizes (4.5) are used in the numerical tests. ClassicalRK4 provides the start vector $v_{1 / 2}$ needed for StaggeredLF4 and StaggeredLF2.

Figure 2 contains convergence results for the $u$-component at two different output times, $t=0.1$ (left) and $t=0.5$ (right). For the $v$-component the results are alike. The marks in the plots correspond with $N=40,80, \ldots, 1280$. The plots are based on efficiency, that is, what we plot are maximum norm global errors (PDE solution minus fully discrete solution) versus computational work (number of integration steps times number of $(f, g)$-evaluations per step times number of spatial grid points).
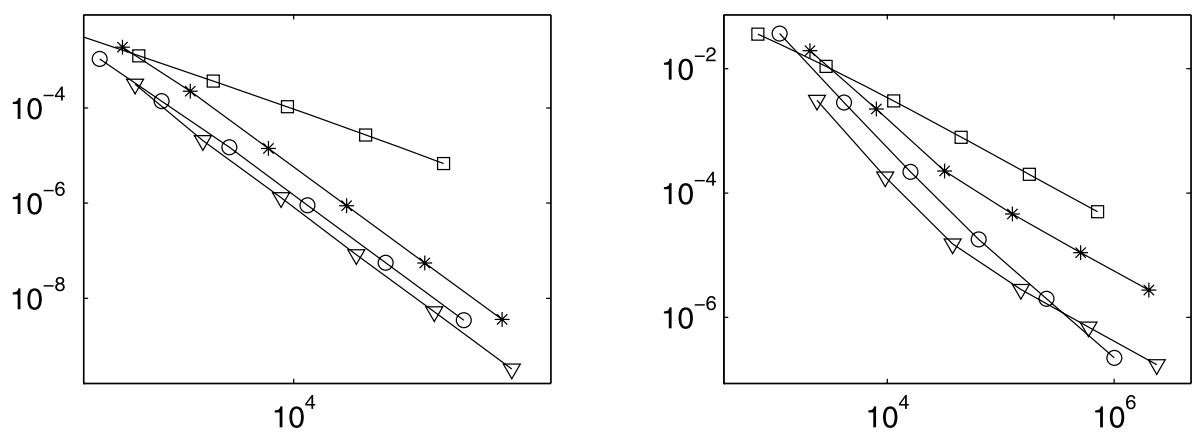

Fig. 2 Loglog convergence plots for problem (4.1). ClassicalRK4 *-marks, StaggeredLF4 o-marks, SymmetricCO4 $\nabla$-marks, StaggeredLF2 $\square$-marks

\footnotetext{
${ }^{1}$ Because the solution consists of outgoing waves and is defined for all $x$, imposing this boundary condition is a consequence of the finite spatial domain and the specific spatial scheme. It does serve our purpose, however, on illustrating the order reduction phenomenon.
} 
At output time $t=0.1$ all three fourth-order methods do converge for $\tau, h \rightarrow 0$ with their ODE order 4, in spite of the prescribed boundary values. So no order reduction occurs. This is due to the fact that at $t=0.1$ the solutions and their derivatives are nearly zero, as can be seen in Fig. 1. Of interest is that both StaggeredLF4 and SymmetricCO4 are more efficient than ClassicalRK4, with a slight advantage for SymmetricCO4. This was not expected, due its higher workload per step and its notably smaller scaled imaginary stability interval compared to StaggeredLF4. Apparently, the symmetric-composition method has smaller error coefficients than the staggered one.

As anticipated, at $t=0.5$ reduction occurs, so that the PDE convergence behavior of the three fourth-order method differs from that at time $t=0.1$. For ClassicalRK 4 and SymmetricCO4 the order goes down to two (the order of StaggeredLF2), whereas for StaggeredLF4 it goes down to three. Due to its order three, in the limit StaggeredLF4 will be most efficient, but it takes a while before it beats SymmetricCO4. On the coarser grids SymmetricCO4 performs best. This is because error terms causing reduction have relatively small error coefficients so that the effect of reduction is normally visible on fine grids only. ${ }^{2}$ This also explains that in spite of the reduction, one is still better off with the higher-order methods than with the simple second-order StaggeredLF2 method. ${ }^{3}$ Finally, note that ClassicalRK4 performs significantly better than StaggeredLF2, but significantly worse than SymmetricCO4 and StaggeredLF4.

\section{Error Analysis for StaggeredLF4}

In this section we will present an error analysis for StaggeredLF4 for arbitrary linear systems

$$
\begin{array}{lll}
u^{\prime}=A v+a(t), & 0<t \leq T, & u(0)=u_{0}, \\
v^{\prime}=B u+b(t), & 0<t \leq T, & v(0)=v_{0},
\end{array}
$$

which explains and gives insight in the order reduction phenomenon. We hereby follow [14], where standard Runge-Kutta methods are discussed (with ClassicalRK4 as a special case). The material from [14] is also contained in [7, Sect. II.2.1]. Essential in the analysis is the Ansatz that $A, B$ and $a(t), b(t)$ are supposed to contain a negative power of a spatial mesh width $h$, and that convergence of numerical approximations is to be understood as convergence towards exact PDE solutions for $\tau, h \rightarrow 0$.

\subsection{Definitions}

For system (5.1), the StaggeredLF4 method (2.3) takes the form

$$
\begin{aligned}
& u_{n+1}=u_{n}+\tau\left(A+\frac{1}{24} \tau^{2} A B A\right) v_{n+1 / 2}+\tau S_{n+1 / 2}^{u}, \\
& v_{n+3 / 2}=v_{n+1 / 2}+\tau\left(B+\frac{1}{24} \tau^{2} B A B\right) u_{n+1}+\tau S_{n+1}^{v},
\end{aligned}
$$

\footnotetext{
${ }^{2}$ In simple cases, like for our test problem, the order reduction can be repaired by transforming the problem to one with a vanishing solution at the boundary [14]. See also [1,3,12] for more involved approaches.

${ }^{3}$ Because of its temporal order two, the fourth-order spatial discretization is a bit of a waste for StaggeredLF2, as fourth-order spatial discretization is more expensive than the most simple second-order one. In this regard, StaggeredLF2 is not equally treated in this comparison. But also with second-order in space it will be less efficient than its three higher-order competitors.
} 
where

$$
\begin{aligned}
& S_{n+1 / 2}^{u}=\frac{1}{24}\left(22 a_{n+1 / 2}+\left(a_{n-1 / 2}+a_{n+3 / 2}\right)+\tau A\left(b_{n+1}-b_{n}\right)+\tau^{2} A B a_{n+1 / 2}\right), \\
& S_{n+1}^{v}=\frac{1}{24}\left(22 b_{n+1}+\left(b_{n}+b_{n+2}\right)+\tau B\left(a_{n+3 / 2}-a_{n+1 / 2}\right)+\tau^{2} B A b_{n+1}\right) .
\end{aligned}
$$

Observe that the forward time-level approximation $u_{n+1}$ is still present in the right hand-side expression for $v_{n+3 / 2}$, and that the inhomogeneous terms $S^{u}$ and $S^{v}$ are taken to be located at $t_{n+1 / 2}$ and $t_{n+1}$, respectively.

Let $u_{h}, v_{h}$ denote a restriction of the PDE solutions approximated by the semi-discrete system (5.1). Substitution into (5.1) defines the local spatial discretization errors $\sigma^{u}$ and $\sigma^{v}$, that is,

$$
\begin{aligned}
& u_{h}^{\prime}(t)=A v_{h}(t)+a(t)+\sigma^{u}(t), \\
& v_{h}^{\prime}(t)=B u_{h}(t)+b(t)+\sigma^{v}(t),
\end{aligned}
$$

and substitution of $u_{h}, v_{h}$ into (5.2) defines local defects $\delta_{n+1 / 2}^{u}$ and $\delta_{n+1}^{v}$, that is,

$$
\begin{aligned}
& u_{h}\left(t_{n+1}\right)=u_{h}\left(t_{n}\right)+\tau\left(A+\frac{1}{24} \tau^{2} A B A\right) v_{h}\left(t_{n+1 / 2}\right)+\tau S_{n+1 / 2}^{u}+\tau \delta_{n+1 / 2}^{u}, \\
& v_{h}\left(t_{n+3 / 2}\right)=v_{h}\left(t_{n+1 / 2}\right)+\tau\left(B+\frac{1}{24} \tau^{2} B A B\right) u_{h}\left(t_{n+1}\right)+\tau S_{n+1}^{v}+\tau \delta_{n+1}^{v} .
\end{aligned}
$$

Next we introduce the full global errors

$$
\varepsilon_{n+1}^{u}=u_{h}\left(t_{n+1}\right)-u_{n+1}, \quad \varepsilon_{n+3 / 2}^{v}=v_{h}\left(t_{n+3 / 2}\right)-v_{n+3 / 2},
$$

and subtract (5.2) from (5.5) for getting the relations defining these global errors in terms of the local error quantities. We find

$$
\left(\begin{array}{c}
\varepsilon_{n+1}^{u} \\
\varepsilon_{n+3 / 2}^{v}
\end{array}\right)=S\left(\begin{array}{c}
\varepsilon_{n}^{u} \\
\varepsilon_{n+1 / 2}^{v}
\end{array}\right)+\tau S_{1}\left(\begin{array}{c}
\delta_{n+1 / 2}^{u} \\
\delta_{n+1}^{v}
\end{array}\right),
$$

where $S=S_{1} S_{2}$ is the amplification operator with

$$
S_{1}=\left(\begin{array}{cc}
I & 0 \\
\tau B+\frac{1}{24} \tau^{3} B A B & I
\end{array}\right), \quad S_{2}=\left(\begin{array}{cc}
I & \tau A+\frac{1}{24} \tau^{3} A B A \\
0 & I
\end{array}\right) .
$$

Regarding the asymptotics $\tau, h \rightarrow 0$, the following general assumptions will be used:

(A1) Stability of (5.7)

(A2) $A=\mathcal{O}\left(h^{-1}\right), B=\mathcal{O}\left(h^{-1}\right), \tau \sim h$

(A3) The PDE solution is sufficiently differentiable

(A4) $A, B, I+\frac{1}{24} \tau^{2} A B, I+\frac{1}{24} \tau^{2} B A$ are inversely bounded

Assumptions (A1), (A2), (A3) are of a general nature. Assumption (A2) makes sense for wave problems like (4.1) ${ }^{4}$ and (A3) justifies Taylor expansions to the level needed in the case

\footnotetext{
${ }^{4}$ For the related problem $\phi_{t}=\psi, \psi_{t}=\phi_{x x}$, assumption (A2) need to be replaced by $A=I, B=$ $\mathcal{O}\left(h^{-2}\right), \tau \sim h$. The order reduction properties discussed here change with this problem.
} 
under consideration. Only assumption (A4) is special, in that it belongs to StaggeredLF4. It will be used in Sect. 5.3. For the numerical test model used above, (A4) can be verified to hold. Below we will prove that if (A1)-(A4) hold, then StaggeredLF4 converges with order 3 instead of its ODE order 4.

\subsection{The Local Errors}

We will first elaborate the local defects introduced in (5.5). Let us write

$$
\delta_{n+1 / 2}^{u}=\delta_{n+1 / 2}^{u, t}+\delta_{n+1 / 2}^{u, s}, \quad \delta_{n+1}^{v}=\delta_{n+1}^{v, t}+\delta_{n+1}^{v, t} \text {, }
$$

where the new upper indices refer to the temporal and spatial defect, respectively. The defects can be determined by eliminating the source term values present in (5.3) through the spatial local error expressions (5.4). The spatial defects are found to be given by

$$
\begin{aligned}
& \delta_{n+1 / 2}^{u, s}=\frac{1}{24}\left(22 \sigma_{n+1 / 2}^{u}+\sigma_{n-1 / 2}^{u}+\sigma_{n+3 / 2}^{u}+\tau A\left(\sigma_{n+1}^{v}-\sigma_{n}^{v}\right)+\tau^{2} A B \sigma_{n+1 / 2}^{u}\right), \\
& \delta_{n+1}^{v, s}=\frac{1}{24}\left(22 \sigma_{n+1}^{v}+\sigma_{n}^{v}+\sigma_{n+2}^{v}+\tau B\left(\sigma_{n+3 / 2}^{u}-\sigma_{n+1 / 2}^{u}\right)+\tau^{2} B A \sigma_{n+1}^{v}\right),
\end{aligned}
$$

and $\delta_{n+1 / 2}^{u, t}$ expanded at $t_{n+1 / 2}$ and $\delta_{n+1}^{v, t}$ expanded at $t_{n+1}$ satisfy

$$
\begin{aligned}
\delta_{n+1 / 2}^{u, t}= & \tau^{4}\left(\frac{1}{1920} u_{h}^{(5)}-\frac{1}{288}\left(u_{h}^{(5)}-A v_{h}^{(4)}\right)-\frac{1}{576} A\left(v_{h}^{(4)}-B u_{h}^{(3)}\right)\right) \\
& +\tau^{6}\left(\frac{1}{322560} u_{h}^{(7)}-\frac{1}{8640}\left(u_{h}^{(7)}-A v_{h}^{(6)}\right)-\frac{1}{46080} A\left(v_{h}^{(6)}-B u_{h}^{(5)}\right)\right) \\
& + \text { h.o.t., } \\
\delta_{n+1}^{v, t}= & \tau^{4}\left(\frac{1}{1920} v_{h}^{(5)}-\frac{1}{288}\left(v_{h}^{(5)}-B u_{h}^{(4)}\right)-\frac{1}{576} B\left(u_{h}^{(4)}-A v_{h}^{(3)}\right)\right) \\
& +\tau^{6}\left(\frac{1}{322560} v_{h}^{(7)}-\frac{1}{8640}\left(v_{h}^{(7)}-B u_{h}^{(6)}\right)-\frac{1}{46080} B\left(u_{h}^{(6)}-A v_{h}^{(5)}\right)\right) \\
& + \text { h.o.t. }
\end{aligned}
$$

From (5.7) follows that the local discretization error, i.e., the error made within a single integration step from the exact solution, is given by

$$
\tau S_{1}\left(\begin{array}{l}
\delta_{n+1 / 2}^{u} \\
\delta_{n+1}^{v}
\end{array}\right)=\tau\left(\begin{array}{cc}
I & 0 \\
\tau B+\frac{1}{24} \tau^{3} B A B & I
\end{array}\right)\left(\begin{array}{c}
\delta_{n+1 / 2}^{u} \\
\delta_{n+1}^{v}
\end{array}\right) .
$$

We now neglect the spatial error contributions (5.11) and (5.12) until further notice. In accordance with the ODE consistency order of 4 , the expansions for the local error then can be seen to begin with $\tau^{5}$-terms. However, this is meaningful only if all terms in the expansion are bounded for $\tau, h \rightarrow 0$. Let us examine the $u$-component (the treatment of $v$ goes identical). Inspection of (5.13) shows that with assumptions (A2), (A3) at hand, the local error satisfies

$$
\tau \delta_{n+1 / 2}^{u, t}=\frac{1}{576} \tau^{5} A B u_{h}^{(3)}+\text { h.o.t. } \sim \tau^{3},
$$


by which we mean that there exist components, which are $\mathcal{O}\left(\tau^{3}\right)$ for $\tau, h \rightarrow 0$, instead of $\mathcal{O}\left(\tau^{5}\right)$. In special cases the order can be higher, but generally it will be three.

To illustrate this, let us consider our test problem (4.1), for which we have $A=B$ and $A=M^{-1} S$, so that $A B u_{h}^{(3)}=M^{-1} S M^{-1} S u_{h}^{(3)}$. The matrices $M$ and $S$ are given by

$$
\begin{gathered}
M=\frac{1}{6}\left(\begin{array}{ccccc}
4 & 1 & & & \\
1 & 4 & 1 & & \\
& \ddots & \ddots & \ddots & \\
& & 1 & 4 & 1 \\
& & & 1 & 4
\end{array}\right), \\
S=\frac{1}{2 h}\left(\begin{array}{ccccc}
0 & 1 & & & \\
-1 & 0 & 1 & & \\
& \ddots & \ddots & \ddots & \\
& & -1 & 0 & 1 \\
& & & -1 & 0
\end{array}\right) .
\end{gathered}
$$

Because at the boundary points $x=0$ and $x=1$ the solution is prescribed, the first rows are truncated at the left and the last rows at the right, as compared with the related matrices

$$
\begin{aligned}
M & =\frac{1}{6}\left(\begin{array}{ccccc}
4 & 1 & & & 1 \\
1 & 4 & 1 & & \\
& \ddots & \ddots & \ddots & \\
& & 1 & 4 & 1 \\
1 & & & 1 & 4
\end{array}\right), \\
S & =\frac{1}{2 h}\left(\begin{array}{ccccc}
0 & 1 & & & -1 \\
-1 & 0 & 1 & & \\
& \ddots & \ddots & \ddots & \\
& & -1 & 0 & 1 \\
1 & & & -1 & 0
\end{array}\right),
\end{aligned}
$$

which we would have in case of periodic boundary conditions. Then, for (5.18), there holds

$$
A B u_{h}^{(3)} \approx\left[\frac{\partial^{5} \phi(h, t)}{\partial t^{3} \partial x^{2}}, \ldots, \frac{\partial^{5} \phi(1-h, t)}{\partial t^{3} \partial x^{2}}\right]^{T}=\mathcal{O}(1),
$$

and this term would cause no reduction. For matrices (5.17), we only get

$$
A B u_{h}^{(3)}=\mathcal{O}(1),
$$

if, in addition to the normal smoothness assumption (A3), the solution derivatives $\phi_{t t t}, \phi_{t t t x}$ and $\phi_{\text {tttxx }}$ would vanish at the boundary points. This follows directly by elaborating the expression

$$
A B u_{h}^{(3)}=M^{-1} S M^{-1} S u_{h}^{(3)} .
$$

If these derivatives don't vanish, the order result (5.16) applies. 
From (5.15) follows that for component $v$ the local error is given by

$$
\left(\tau B+\frac{1}{24} \tau^{3} B A B\right) \tau \delta_{n+1 / 2}^{u, t}+\tau \delta_{n+1}^{v, t} .
$$

With assumption (A2) at hand, it is seen immediately that in general $v$ suffers from the same level of order reduction as $u$.

Finally, with the same reasoning, one can deduce from (5.11) and (5.12) that the spatial contributions to the full local errors are of the order of the local spatial discretization errors $\sigma^{u}, \sigma^{v}$ introduced in (5.4). Hence the spatial order behavior is regular.

\subsection{The Global Errors}

The numerical tests presented in Sect. 4 revealed a PDE convergence order 3 for StaggeredLF4. However, we also found its local error to behave according to this order. This is contradictory to the standard estimation technique of transferring local to global errors through recursion (5.7) and assumption (A1) given in (5.9), since this would result in a PDE convergence order 2. Fortunately, very often the reduction is one unit less for the global error, due to cancellation effects. This happens also for StaggeredLF4. Below we will prove that the method converges with order 3 assuming (A1)-(A4).

To take the cancellation into account, we will use Lemma II.2.3 from [7]. Denote (5.7) by $\varepsilon_{n+1}=S \varepsilon_{n}+\tau \delta_{n}$. The idea of the lemma is to assume that $\delta_{n}$ can be written as

$$
\begin{aligned}
& \delta_{n}=(I-S) \xi_{n}+\eta_{n}, \quad \text { such that, } \\
& \xi_{n} \sim \tau^{2}, \quad \xi_{n+1}-\xi_{n} \sim \tau^{3}, \quad \eta_{n} \sim \tau^{3} .
\end{aligned}
$$

The difference $\hat{\varepsilon}_{n}=\varepsilon_{n}-\tau \xi_{n}$ then satisfies $\hat{\varepsilon}_{n+1}=S \hat{\varepsilon}_{n}+\tau \hat{\delta}_{n}, \hat{\delta}_{n}=\eta_{n}-\left(\xi_{n+1}-\xi_{n}\right)$, for which the standard estimation technique of 'summing up all local errors' then can be used to prove PDE convergence order 3.

As shown above we may neglect the spatial contributions (5.11) and (5.12) to the local error, and we will do so for convenience of presentation. First, from (5.8) we write $S$ as

$$
S=\left(\begin{array}{cc}
I & S_{12} \\
S_{21} & I+S_{21} S_{12}
\end{array}\right), \quad \begin{aligned}
& S_{12}=\tau A+\frac{1}{24} \tau^{3} A B A, \\
& S_{21}=\tau B+\frac{1}{24} \tau^{3} B A B .
\end{aligned}
$$

Likewise, from (5.15) we write $\delta_{n}$ as

$$
\delta_{n}=\left(\begin{array}{c}
\delta_{n+1 / 2}^{u} \\
\delta_{n+1}^{v}+S_{21} \delta_{n+1 / 2}^{u}
\end{array}\right) .
$$

Next we choose $\eta_{n}=0$ and compute

$$
\begin{aligned}
\xi_{n} & =(I-S)^{-1} \delta_{n}=\left(\begin{array}{l}
-S_{21}^{-1} \delta_{n+1}^{v} \\
-S_{12}^{-1} \delta_{n+1 / 2}^{u}
\end{array}\right) \\
& =\left(\begin{array}{l}
-\left(I+\frac{1}{24} \tau^{2} A B\right)^{-1} B^{-1}\left(\tau^{-1} \delta_{n+1}^{v}\right) \\
-\left(I+\frac{1}{24} \tau^{2} B A\right)^{-1} A^{-1}\left(\tau^{-1} \delta_{n+1 / 2}^{u}\right)
\end{array}\right),
\end{aligned}
$$


where we have used assumption (A4) given in (5.9).

From (5.21) then follows, that, for proving the order 3 convergence, there remains to assess that

$$
\xi_{n} \sim \tau^{2}
$$

Consider expression (5.13) for $\delta_{n+1 / 2}^{u, t}$ and its order reducing term $A B u_{h}^{(3)}$ given in (5.16). By the multiplication with the inverse of $A$, we gain one order for this term. By the division by $\tau$, we loose one. Consequently, with assumption (A4), the contribution of this term to $\xi_{n}$ is just $\sim \tau^{2}$. All other terms are of higher order in $\tau$, meaning that for the second component of $\xi_{n}$ the proof is complete. The proof for the first component of $\xi_{n}$ goes in the same way, using similar arguments for $\delta_{n+1}^{v, t}$.

\section{Final Remarks and Conclusions}

The numerical literature on partial differential equations shows a development towards higher-order discretization methods. In line with this development, the current paper was devoted to higher-order, explicit time integration of the partitioned system (1.1) emanating from wave-type problems. We have discussed and compared three fourth-order methods, including StaggeredLF4 - the time-staggered method (2.3), SymmetricCO4-the symmetriccomposition method (3.5) derived from symplectic Euler, and ClassicalRK4-the classical Runge-Kutta method. In particular our aim was to assess the merits of StaggeredLF4, which was proposed in $[4,5]$.

No doubt this method is a very valuable contribution to the field. It is significantly more efficient than ClassicalRK4 and the classical, second-order, staggered leapfrog method (2.1). Like most higher-order multi-stage methods, the fourth-order method can suffer from order reduction, but only by one unit of convergence order, and it is felt only on rather fine grids. Its strength lies in its scaled imaginary stability boundary, which is considerably larger than for its two fourth-order competitors, see (3.8).

On the other hand, our numerical comparison with SymmetricCO4, although limited, indicates that symmetric composition based on symplectic Euler is nearly as efficient as time staggering, if not more efficient. In a sequel to this paper we therefore plan to report on more extensive research to the benefits of high-order composition for wave-type partial differential equations with a focus on systems (1.1), including systems of the form $u^{\prime}=v, v^{\prime}=g(t, u)$, since this gives more room for nonlinear second-order problems, and possibly other partitioned semi-discrete forms.

A further bonus of SymmetricCO4 is that it is based on symplectic Euler and thus it is also symplectic. This is a valuable property not shared by StaggeredLF4. To illustrate this we conclude the paper with numerically comparing SymmetricCO4 and StaggeredLF4 for the Kepler two-body problem

$$
\ddot{q}_{1}=-\frac{q_{1}}{\left(q_{1}^{2}+q_{2}^{2}\right)^{3 / 2}}, \quad \ddot{q}_{2}=-\frac{q_{2}}{\left(q_{1}^{2}+q_{2}^{2}\right)^{3 / 2}} .
$$

Copying data from [6], it was solved for the initial values $q_{1}(0)=1-e, q_{2}(0)=0$, $\dot{q}_{1}(0)=0, \dot{q}_{2}(0)=\sqrt{(1+e) /(1-e)}$ with eccentricity $e=0.6$. Writing it in first-order form gives a 4-component partitioned system of type (1.1). The solution is $2 \pi$-periodic and was computed over 100 periods, using $\tau=\pi / 20$ for StaggeredLF4 and $\tau=\pi / 16$ for SymmetricCO 4 for equal expense per time step. The missing initial value $v_{1 / 2}$ for StaggeredLF4 was 

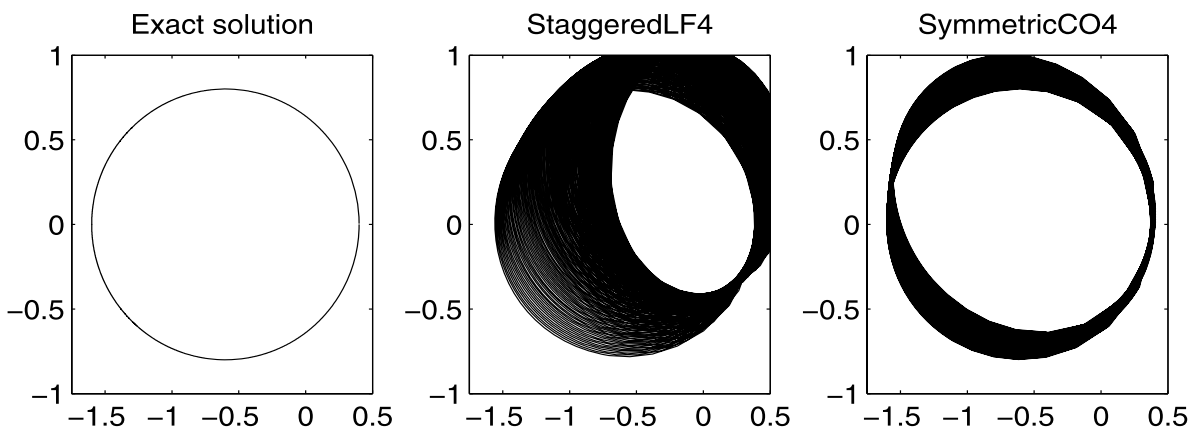

Fig. 3 Exact and numerical solutions of the Kepler problem $\left(q_{2}\right.$ versus $\left.q_{1}\right)$ over 100 periods

here computed with SymmetricCO4. Figure 3 shows that SymmetricCO4 is indeed more accurate than StaggeredLF4, which in turn is significantly more accurate than ClassicalRK4 (not shown here).

\section{References}

1. Abarbanel, S., Gottlieb, D., Carpenter, M.H.: On the removal of boundary errors caused by Runge-Kutta integration of nonlinear partial differential equations. SIAM J. Sci. Comput. 17, 777-782 (1996)

2. Blanes, S., Moan, P.C.: Practical symplectic partitioned Runge-Kutta and Runge-Kutta-Nyström methods. J. Comput. Appl. Math. 142, 313-330 (2002)

3. Calvo, M.P., Palencia, C.: Avoiding the order reduction of Runge-Kutta methods for linear initialboundary value problems. Math. Comput. 71, 1529-1543 (2002)

4. Ghrist, M.L.: High-order finite difference methods for wave equations. Ph.D. Thesis, University of Colorado, Boulder, USA (2000)

5. Ghrist, M., Fornberg, B., Driscoll, T.A.: Staggered time integrations for wave equations. SIAM. J. Numer. Anal. 38, 718-741 (2000)

6. Hairer, E., Lubich, C., Wanner, G.: Geometric Numerical Integration. Springer Series in Computational Mathematics, vol. 31. Springer, New York (2002)

7. Hundsdorfer, W., Verwer, J.G.: Numerical Solution of Time-Dependent Advection-Diffusion-Reaction Equations. Springer Series in Computational Mathematics, vol. 33. Springer, New York (2003)

8. Kinnmark, I.P.E., Gray, W.G.: One-step integration methods of third-fourth order accuracy with large hyperbolic stability limits. Math. Comput. Simul. 18, 181-184 (1984)

9. McLachlan, R.I.: On the numerical integration of ordinary differential equations by symmetric composition methods. SIAM J. Sci. Comput. 16, 151-168 (1995)

10. McLachlan, R.I., Quispel, G.R.W.: Splitting methods. Acta Numer. 11, 341-434 (2002)

11. Murua, A., Sanz-Serna, J.M.: Order conditions for numerical integrators obtained by composing simpler integrators. Philos. Trans. Roy. Soc. A 357, 1079-1100 (1999)

12. Pathria, D.: The correct formulation of intermediate boundary conditions for Runge-Kutta time integration of initial boundary value problems. SIAM J. Sci. Comput. 18, 1255-1266 (1997)

13. Sanz-Serna, J.M., Calvo, M.P.: Numerical Hamiltonian Problems. Chapman \& Hall, London (1994)

14. Sanz-Serna, J.M., Verwer, J.G., Hundsdorfer, W.H.: Convergence and order reduction of Runge-Kutta schemes applied to evolutionary problems in partial differential equations. Numer. Math. 50, 405-418 (1987)

15. Sonneveld, P., van Leer, B.: A minimax problem along the imaginary axis. Nieuw Archief Wiskd. 3, 19-22 (1985)

16. van der Houwen, P.J.: Construction of Integration Formulas for Initial Value Problems. North-Holland, Amsterdam (1977)

17. Yee, K.S.: Numerical solution of initial boundary value problems involving Maxwell's equations in isotropic media. IEEE Trans. Antennas Propag. 14, 302 (1966) 